\title{
Vorwort zur vierten Auflage.
}

(1904.)

In den wenigen Jahren, welche seit der letzten Auflage dieses Buches verflossen sind, hat sich in den Auffassungen auf kriminalistischem Gebiete mehr geändert, als dies sonst in Jahrzehnten der Fall war. Schon lange als drängend empfunden, haben sich diese Änderungen nun mit Gewalt geltend gemacht und so wankt auf unserem Gebiete alles, überall wollen neue Anschauungen zum Durchbruche gelangen. Kaum einer der Grundsätze des alten Strafrechts steht mehr fest, die Fragen nach Umwertung der Vorstellungen über Determination, Verantwortung und Zurechnung drängen in das Strafgesetz, ganze Kapitel sollen davon ausgeschieden, andere völlig umgeändert werden, ein neues Strafensystem muB erdacht, der Strafzweck fixiert und eine Fürsorgeordnung eingeführt werden. Kaum weniger Bewegung herrscht im StrafprozeB; unsere alten Anschauungen über den Beweis halten nicht mehr, das Vertrauen auf die wichtigsten Beweismittel, Zeugenaussagen und richterliche Anschauung sind erschüttert, neue Beweismittel, die Realien des Strafrechts, machen sich mit Nachdruck geltend und neue Theorien darüber, was als wahr anzusehen ist, wollen geprüft werden. Das Laienelement in der Rechtsprechung, in das man noch vor wenigen Jahrzehnten alles Heil verlegt hatte, brachte uns nur Schwierigkeiten, Unwahrheit und unabsehbare Gefahren, und wer die Frage ehrlich überlegt, zweifelt nur mehr darüber, wi e wir dieses Unheil wieder los werden könnten.

Ebenso unsicher ist die Auffassung über das Vorverfahren, die Stellung der Staatsanwaltschaft und der Verteidigung, über die Rechtsmittel geworden - kurz, wir befinden uns in einem so stürmischen Gärungsprozesse, daß wir einerseits mit Freude über das überall pulsierende Leben, anderseits mit Bedenken über die Schwierigkeit dieser Lösungen an die Arbeit gehen.

Aber auch auf unserem besonderen Gebiete herrscht das regste Leben. Die Kriminalistik hat sich emporgearbeitet, überall regen sich fleißige Hände um ihr Gebiet zu bebauen, viele ihrer Fragen wurden zur Lösung gebracht, neue Fragen angeregt, wirkliche Probleme sind entstanden und der Nutzen war ein tausendfältiger. -

Mit besonderer Befriedigung darf auf die Entwickelung der subjektiven Kriminalpsychologie geblickt werden, der Psychologie des 
Zeugen, Sachverständigen und Richters. Auf ihre Wichtigkeit wurde zuerst in der ersten Auflage dieses Handbuches, also vor 12 Jahren, hingewiesen; dort wurde das Trügerische und Gefährliche namentlich der Zeugenaussagen entwickelt und die Notwendigkeit ihrer Unterstützung, Prüfung und teilweise Ersetzung durch die Realien des Strafrechts dargetan. Später wurde dieses Problem von mir („Kriminalpsychologie“ Graz 1898) besonders behandelt, dann von mehrfacher Seite aufgegriffen und heute beschäftigt sich ein ganzes periodisches Unternehmen (,Beiträge zur Psychologie der Zeugenaussage“" Lpzg., J. A. Barth) ausschlieBlich und in verdienstlicher Weise mit dieser wichtigen Frage. -

Die Stellung der Kriminalistik im System (s. die Tabelle am Schlusse dieses Vorwortes) ist eine andere geworden, als vom Anfange an richtig geschienen hat; als Lehrgegenstand soll sie nicht vereinzelt dastehen, sie muB auf breite Grundlage gestellt werden; neben ihr haben sich Schwesterdisziplinen: Kriminalanthropologie, Kriminalpsychologie, Kriminalsoziologie und Kriminalistik so kräftig emporgearbeitet, daB sie unbedingte Beachtung verdienen; sie sind aber alle mit der Kriminalistik an allen ihren Grenzen so innig verbunden, daB sich keine dieser Disziplinen allein bewegen kann, sie alle streben zusammen vorwärts, sie können sich auch nur zugleich entwickeln. So hat sich die Notwendigkeit ergeben, aus den ge. nanten Disziplinen, also: Kriminalistik, Kriminalanthropologie, Kriminalpsychologie, Kriminalsoziologie und Kriminalstatistikeine fest gegliederte und fest vereinte Gruppe zubilden, sie müssen als strafrechtliche Hilfswissenschaften gemeinsame Pflege erhalten und sich gemeinsam, bescheiden, aber unabweislich, in den Dienst des Strafrechts stellen. Wir haben die strafrechtlichen Hilfswissenschaften über ihre ersten Anfänge hinausgebracht, heute erklären wir aber mit Nachdruck, daß ohne ihre Kenntnis ein Kriminalist weder in Theorie noch in Praxis ausgebildet erscheint, ihre unentbehrliche Notwendigkeit kann nicht mehr bezweifelt werden. -

Was den Stoff der vorliegenden neuen Auflage anlangt, so wurden zwar keine neuen Kapitel eingefügt und keine früheren weggelassen - was zum Materiale gehört, ist längst klar geworden. Wohl aber mußte fast alles geändert, erweitert, gekürzt und ergänzt und so viel neue Literatur eingefügt werden, daB die neue Auflage als völlig umgearbeitet bezeichnet werden kann.

Die äuBere Form des Buches liegt diesmal in zwei Bänden vor, was zum Teile durch die Vermehrung des Inhalts, zum Teil durch deutlicheren Druck, zum Teil durch das Bestreben nach größerer Handlichkeit veranlaßt wurde. 
Dem Inhalte nach erscheint das Werk derzeit zum letzten Male in dieser Anordnung. Als die Kriminalistik zuerst in die Welt trat, konnte begreiflicher, oder wenigstens entschuldbarermaBen noch nicht klar sein, welche Materien zu ihr gehören und in welcher Gruppierung diese zu bieten seien. Im Laufe der Arbeit ergab es sich aber, daB in nicht weiter zu vertretender Weise in dieser Disziplin eine rein theoretische und eine rein praktische Lehre vereinigt und vermengt sei.

Dereine Teil befaßt sich mit der Erscheinung des Ver. brechens in objektiver Form; allgemeine Erscheinungen; das Wesen der Verbrecher, ihre Tricks, ihre Sprache, ihre Zeichen, ihre Verständigungsmittel, Simulationen, Täuschungsmittel, Lügen (normales und pathoformes), falsche Namen, Gewohnheiten, Aberglauben usw.; bes ondere Erscheinungen bei den einzelnen Verbrechen, namentlich: Diebstahl, Betrug, Fälschungen, Mord, Abtreibung, Brandlegung usw. Endlich: überall nach Tunlichkeit die $h$ is toris che Entwicklung.

Der zweite Teil enthält lediglich praktische Anweisungen; über das Vorgehen des UR., seine Heranbildung, das Verhör, die auswärtigen Amtshandlungen, den Verkehr mit den Sachverständigen und ihre Verwendung, den praktischen Teil der Gaunerpraktiken, die Waffen, die verschiedenen Spuren, die Fertigkeiten und Techniken des UR. usw. - endlich die praktischen Fragen bei den einzelnen Delikten.

Es wird somit, sollte eine neue Auflage noch nötig werden, das Buch erscheinen als

System der Kriminalistik.

I. Teil: Theoretische Erscheinungslehre des Verbrechens.

II. Teil : Praktische Untersuchungskunde.

Eine integrierende Ergänzung dieses Buches bildet das nunmehr im XIV. Bande stehende, von mir herausgegebene „A rchiv für Kriminal-Anthropologie und Kriminalistik" (Leipzig, F. C. W. Vogel), welches den Kontakt unserer Disziplin mit dem stets neu schaffenden Leben herzustellen bestimmt ist. Die Kriminalistik strebt als propulsive und stetig sich erweiternde Lehre nach Expansion und Ausbreitung auf neues Material, das ihr durch dieses Archiv zugeführt werden soll. - 
Zur Klärung darüber, wie ich mir die Stellung der Kriminalistik im System des Strafrechts denke, füge ich endlich ein Tableau über die Gesamtlehre bei :

\section{Kriminologie}

als die Lehre vom Verbrechen:

Allgemeine Erscheinungslehre des Verbrechens

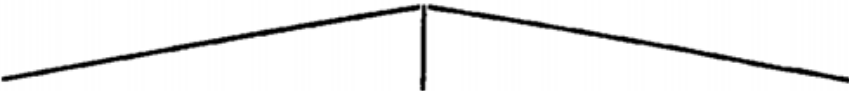

Krim. Anthropologie Krim. Soziologie Besondere Erscheinungslehre<smiles>C[13CH2]</smiles>

Krim.

logie

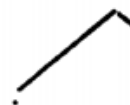

Krim.

Krim. Statistik Kr. Psycho-

Soziale logie

Psychologie

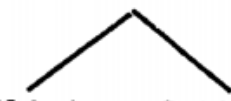

Krimi- Subjekt. nalistik Krim.

Psychologie

Kriminalpolitik

Materielles und formelles Strafrecht

Prag, Neujahr 1904.

Hans Groß. 


\section{Vorwort zur fünften Auflage.}

(1907.)

Auch die neue Auflage ist vollständig umgearbeitet und bis auf den letzten Stand der Fragen gebracht; sie hat auch einige neue Kapitel erhalten („nächste Umgebung des Körpers“, „Linkshändigkeit", „Selbstmord“, „Kindmord“). -

Die Voraussagung des Vorwortes der vierten Auflage: das Werk werde künftig in völlig anderer Anordnung erscheinen, ist nicht zugetroffen; ich habe die frühere Einteilung des Stoffes beibehalten, da sich die Kritik und treue, alte Freunde des Buches einstimmig gegen eine Umgestaltung aussprachen und verlangten, es solle die bewährte, eingewöhnte und gebilligte Anordnung belassen werden. Dieser, in der vierten Auflage erwähnte Plan ist daher endgültig aufgegeben.

Eine äußere Änderung erfuhr das Buch dahin, daB es nicht mehr in zwei Bänden, sondern in zwei Teilen (mit fortlaufender Paginierung) erscheint, um das Aufsuchen nach dem Register zu erleichtern.

Bezüglich des Inhalts berufe ich mich lediglich auf das, in den Vorworten zur dritten und vierten Auflage Gesagte; ich habe nichts hinzuzufügen. -

Der Stoff der Kriminalistik ist derart angewachsen, daB er in einem Buche allein nicht mehr Raum findet, und es ist daher das von mir herausgegebene „Archiv für Kriminalanthropologie und Kriminalistik", (jetzt im 28. Bande) Lpzg., F. C. W. Vogel, wie schon im Vorworte zur 4. Aufl. gesagt, ein integrierender Bestandteil des vorliegenden Buches geworden.

Ich danke abermals der so überaus wohlwollenden Kritik, des In- und Auslandes, allen Übersetzern (das Werk ist nunmehr in fast alle Kultursprachen übersetzt), allen Freunden des Buches und seinen Helfern; endlich auch der Verlagsanstalt für den schönen, bequemen Druck, die Aufnahme vieler neuer Abbildungen und ihr vielfach bewiesenes Entgegenkommen bei der mühsamen Herstellung der neuen Auflage.

Graz, Spätherbst 1907.

Hans Groß. 
